\title{
Problems Associated with Grid Convergence of Functionals
}

\author{
Manuel D. Salas ${ }^{1}$ and Harold L. Atkins ${ }^{2}$ \\ 1 NASA Langley Research Center m.d.salas@nasa.gov \\ 2 NASA Langley Research Center harold.1.atkins@nasa.gov
}

Keywords: Code verification, grid convergence, supersonic blunt-body, drag functional.

Summary. The current use of functionals to evaluate order-of-convergence of a numerical scheme can lead to incorrect values. The problem comes about because of interplay between the errors from the evaluation of the functional, e.g., quadrature error, and from the numerical scheme discretization. Alternative procedures for deducing the order-property of a scheme are presented. The problem is studied within the context of the inviscid supersonic flow over a blunt body; however, the problem and solutions presented are not unique to this example.

\section{Introduction}

Computational Aerodynamicists conduct most of their grid convergence studies by studying the behavior of solution functionals, e.g., drag, lift and moment coefficients, as the computational grids are refined. Functionals are used for several reasons: first, their accurate evaluation is of intrinsic value; and second, they provide a means of determining convergence properties of a numerical scheme without looking directly at hundreds of thousands of field point values. Ideally, an error measure should be used to examine order-properties of grid convergence studies; however, exact solutions are usually not available for flows of practical interest. Therefore, estimating convergence properties using functionals is frequently the only course of action available.

However, there are some subtle problems associated with the use of functionals for grid convergence studies, and if these problems are not recognized and resolved, the results that follow from the use of functionals can be very misleading. It is the purpose of this paper to expose these problems, and where possible, suggest solutions.

There are many aspects of a numerical order-properties analysis that must be done correctly in order for the analysis to be reliable. Paramount among 
these are: that grid refinements must be uniform, preferably with grids sequences that are hierarchical; and the iterative methods used to solve the discrete equations on a given grid must be sufficiently converged, preferably with residuals reduced several orders of magnitude below the solution error. Of course, this is complicated by the fact that the errors are not known a priori.

The problems that are associated with the use of functionals for the study of grid convergence rates are illustrated with numerical results from the computation of a blunt-body in an inviscid supersonic stream. However, it should be emphasized that the problems discussed are not unique to the blunt-body problem, indeed they are not unique to fluid mechanics, and may occur in any grid convergence study involving functionals. The particular case studied is the Mach 6 flow of an inviscid gas over a circular cylinder. In the numerical implementation the problem is solved as a time dependent problem with the bow shock wave fitted as a boundary of the flow. By fitting the shock, the numerical scheme acts only on a smooth flow region. Thus, the computation is limited to the layer bounded by the bow shock, the circular cylinder, the symmetry line, and a supersonic outflow boundary imposed at some $\theta=\theta_{\max }$, see Fig. 1.

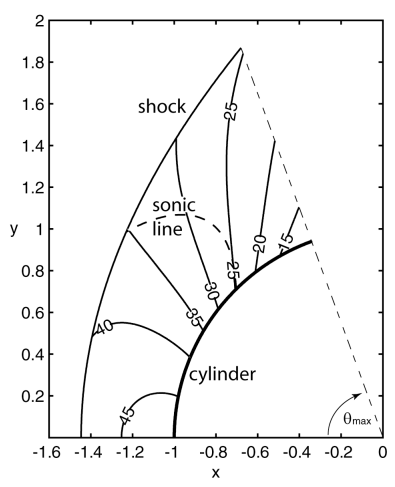

Fig. 1. Supersonic blunt-body flow field, showing isobars, $M_{\infty}=6$.

The physical plane is transformed to a computational plane where $\mathrm{N}$ and $\mathrm{M}$ mesh points are uniformly distributed between the body and the shock and between the symmetry line and the outflow line, respectively. The predictor/corrector MacCormack scheme [1] is used for the numerical integration of the Euler equations.

Table 1 shows results obtained with a series of grids. The number of mesh points corresponding to each $k^{t h}$ grid are $\mathrm{N}_{k}=3 \times 2^{k}$ and $\mathrm{M}_{k}=5 \times 2^{k}$. Columns 2 and 3 display the inviscid drag coefficient computed with the trapezoidal rule (TR) and with Simpson's rule (SR). The drag coefficient 
order-of-convergence is given by

$$
p_{k}=\log _{2}\left[\frac{C_{d, k}-C_{d, k+1}}{C_{d, k+1}-C_{d, k+2}}\right],
$$

and is shown in the last two columns. The order-of convergence for $k=3$ for TR and SR shows a large discrepancy and both results are significantly greater than the formal order of the scheme which is second order. For $k=4$, the drag coefficient is not monotone and the order-of-convergence evaluation fails. (Note that the order-of-convergence for grid $k$ depends on the solutions from grids $k, k+1$, and $k+2$ ).

Table 1. $M_{\infty}=6$ cases investigated. For the three finest grids the trapezoidal rule (TR) computed drag is not monotone and for both quadrature rules the computed drag exhibits super-convergence.

\begin{tabular}{lllll}
\hline$k$ & $C_{d}(\mathrm{TR})$ & $C_{d}(\mathrm{SR})$ & $p(\mathrm{TR})$ & $p(\mathrm{SR})$ \\
\hline 1 & 1.8755919 & 1.8767669 & 1.92 & 1.90 \\
2 & 1.8706109 & 1.8709412 & 2.73 & 2.58 \\
3 & 1.8692925 & 1.8693766 & 4.03 & 3.25 \\
4 & 1.8690942 & 1.8691147 & - & - \\
5 & 1.8690821 & 1.8690872 & & \\
6 & 1.8690859 & 1.8690872 & & \\
\hline
\end{tabular}

To establish that there is reason to suspect these results, consider the behavior of the error norm in total temperature. For this problem in the steady state, the total enthalpy, and hence the total temperature, is constant. The $L_{2}$ and $L_{\infty}$ norms of the total temperature error are shown in Fig. 2. The orderof-convergence based on the $L_{2}$ and $L_{\infty}$ norms is 2.03 and 1.84, respectively. These are in fairly good agreement with the formal order of the scheme. Why then is the order-of-convergence of the drag functional misbehaving?

\section{Order-of-convergence of functionals}

To answer the last question, first consider the following question: If the surface pressure converges with order $p$, what should the expected order-ofconvergence of the drag-functional be? To this end, let the computed surface pressure, $P_{c}$, normalized by the free stream pressure, $P_{\infty}$, be given by $P_{c} / P_{\infty}=P_{e} / P_{\infty}+\alpha(\theta) h^{p}$, where $P_{e}$ is the exact surface pressure. The sectional drag coefficient is defined by

$$
C_{d}=\int_{0}^{\theta_{\max }}\left(P_{c} / P_{\infty}-1\right) b(\theta) \cos (\theta) d \theta /\left(\frac{1}{2} \gamma M_{\infty}^{2} A_{r e f}\right)
$$




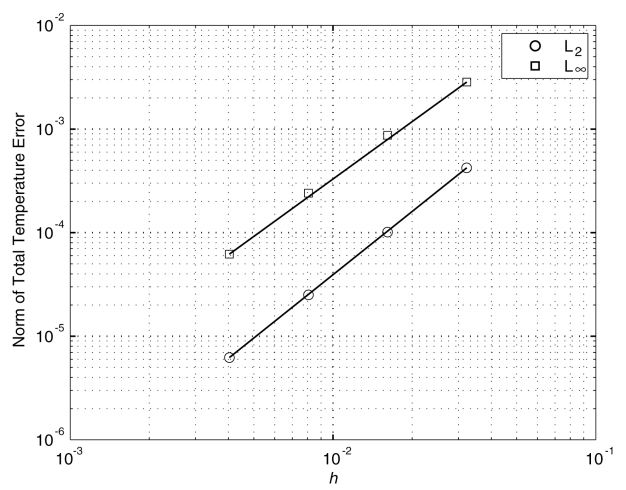

Fig. 2. $L_{2}$ and $L_{\infty}$ of total temperature error for entire shock layer, based on results from grids $k=3,4,5$, with $h_{k}=1 / \sqrt{N_{k} M_{k}}$.

where $\gamma$ is the ratio of specific heats, $M_{\infty}$ is the free stream Mach number, $b(\theta)$ is the body radius, and $A_{\text {ref }}$ is a reference surface area, here taken as the projected plan-form area. Therefore, $C_{d}=C_{d, e}+\Gamma h^{p}$ where $\Gamma$ is a constant and $C_{d, e}$ is the exact value of the drag coefficient; therefore, the drag-functional should converge with order $p$.

\section{The problem with quadrature}

If it is assumed that the pressure order-of-convergence behaves like the total temperature order-of-convergence, then the result just obtained for drag is not consistent with the results of Table 1 . The problem lies in the numerical integration of (2). The integration is approximated by a quadrature taken over $\mathrm{M}$ equally spaced intervals on the surface of the cylinder, i.e. $\int_{0}^{\theta_{\max }} f d \theta \approx$ $\sum_{1}^{\mathrm{M}+1} a_{i} f_{i}$. The quadrature has a leading error of order $h^{q}$. It is easy to show that $C_{d}=C_{d, e}+\beta_{q} h^{q}+\Gamma h^{p}+O\left(h^{q+p}\right)$, where $\beta_{q}$ is a constant and $q$ equals 2 for TR and 4 for SR. Using this relation for $C_{d}$ in (1) we find

$$
\bar{p}=p+\log _{2}\left\{\frac{2^{p-2} \beta_{q}\left[1-2^{q}\right]+h^{p-q} \Gamma\left[1-2^{p}\right]}{2^{2(p-2)} \beta_{q}\left[1-2^{q}\right]+h^{p-q} \Gamma\left[1-2^{p}\right]}\right\},
$$

where $h$ is the coarse grid spacing. Here $\bar{p}$ is the computed order-of-convergence and the $\log _{2}$-term is an error brought about by the interplay between the quadrature error and algorithmic error. In the limit $h \rightarrow 0$, the $\bar{p}$ behavior is given by $\bar{p} \rightarrow q$ if $p \geq q$, and $\bar{p} \rightarrow p$ if $p<q$. However, in a computation $h$ will always be finite and having $p<q$ is not a guarantee that the $\log _{2}$-term will be small. 


\section{How to eliminate the quadrature error}

A solution to the quadrature problem can be found by studying (1). Consider the numerator. The numerator is the difference between the drag coefficients of the medium $(\mathrm{k}+1)$ and coarse $(\mathrm{k})$ grids. The medium grid has a quadrature error of order $(h / 2)^{2}$, while the coarse grid has a quadrature error of order $h^{2}$. These errors do not cancel out and their interplay with the algorithmic error causes some (not all) of the problems in the results of Table 1. The solution is to implement the quadrature in such a way that the quadrature errors of the medium and coarse grids cancel. To do this, evaluate both the medium and coarse grid quadratures using an $h$ interval, i.e., use only every other point of the medium grid. The same idea is applied to the denominator by evaluating both the medium and fine grid quadratures using an $h / 2$ interval.

\section{Higher-order algorithmic error model}

The actual algorithmic error in any numerical solution on any given grid contains a full hierarchy of errors that are unknown, but are generally assumed to be of the form $u_{c, k}=u_{e}+\sum_{n=p}^{\infty} \alpha_{n} h_{k}^{n}$, where $p$ is the unknown actual order of the numerical method. The standard method for deducing order properties from grid convergence, described earlier, is obtained by fitting a single error mode of the form $u_{c, k}=u_{e}+\alpha h_{k}^{p}$ to the actual error. For sufficiently small $h$, the actual error is dominated by the lowest order term, and the single mode model provides a good fit and an accurate prediction of the order-of-convergence. However, for larger $h$ above this asymptotic region (it is surprising how small $h$ has to be to reach asymptotic convergence), multiple error modes are competing, and their projection onto a single mode can be erroneous. Consider then a two-mode error model:

$$
C_{d, k}=C_{d, e x}+\alpha_{1} h_{k}^{p}+\alpha_{2} h_{k}^{p+1} .
$$

For this model, using a sequence of four grids ( $\mathrm{k}$ through $\mathrm{k}-3$ ), where $h_{k} / h_{k-1}=h_{k-1} / h_{k-2}=h_{k-2} / h_{k-3}=2$, we find the order-of-convergence to be

$$
p=\log _{2}\left[\frac{3 \Delta_{k-2, k-1}-\sqrt{9 \Delta_{k-2, k-1}^{2}+8 \Delta_{k-1, k} \Delta_{k-2, k-3}}}{4 \Delta_{k-1, k}}\right]
$$

where $\Delta_{i, j}=C_{d, i}-C_{d, j}$. It is important to monitor the ratio $\alpha_{2} h / \alpha_{1}$.

$$
\frac{\alpha_{2} h}{\alpha_{1}}=\frac{4\left(1-2^{p}\right)}{\left(1-2^{p+1}\right)} \frac{\left(2^{p} \Delta_{k-2, k-1}-\Delta_{k-1, k}\right)}{\left(\Delta_{k-2, k}-2^{p+1} \Delta_{k-2, k-1}\right)}
$$

When this ratio is less than one, the one-mode error model is valid. With a two-mode error model and eliminating the quadrature error as previously described, we obtain the results listed in Table 2. For more details see [2]. 
Table 2. Drag order-of-convergence using higher order method and coarse grid interval, $k=3$, for quadrature rules.

\begin{tabular}{lllll}
\hline$k$ & $C_{d}(\mathrm{TR})$ & $C_{d}(\mathrm{SR})$ & $p(\mathrm{TR})$ & $p(\mathrm{SR})$ \\
\hline 3 & 1.8692925 & 1.8693766 & 1.674 & 1.681 \\
\hline 4 & 1.8690328 & 1.8691145 & & \\
\hline 5 & 1.8690056 & 1.8690872 & & \\
\hline 6 & 1.8690056 & 1.8690871 & & \\
\hline \hline
\end{tabular}

\section{Conclusions and recommendations}

With the increased reliance in both science and engineering on the numerical solution of partial differential equations, the subject of code verification has become increasingly significant. An important element of code verification is the study of grid convergence. Most studies today of this subject have been at best superficial and in many cases painfully inadequate. This paper is an attempt to reverse this trend by first highlighting a series of problems that exist in the standard order-of-convergence analysis, particularly as it relates to the evaluation of functionals, and second by providing a number of solutions and workarounds to these problems. It is important to distinguish between a code verification effort and an effort to determine if a particular solution to a specific problem is sufficiently accurate for some intended use. The two tasks are very different. A rigorous grid convergence and order-of-convergence study can aid in determining if an algorithm has been implemented correctly. However, such a rigorous study requires grids of the same family and grid refinements that are uniform, preferably with grids sequences that are nested. In the second task, limited time and resources often lead to compromising one or more attributes of a rigorous study. While non-uniform mesh refinement may lead to some improvement in the solution, order-of-convergence properties computed from non-uniform refinements or ill-converged solutions sets are meaningless. Whenever possible, error norms should be used to establish the order-of-convergence. The higher order analysis developed in Sect. 5 is the best way to evaluate if the asymptotic range has been reached or if more levels of grid refinement are needed to reach it. It should be part of any rigorous grid convergence study. Reference [2] provides a more in depth study of these issues.

\section{References}

1. MacCormack, RW. The effect of viscosity in hypervelocity impact cratering. AIAA Paper No. 69-354, 1969.

2. Salas, MD and Atkins, HL. On problems associated with grid convergence of functionals. Comp. \& Fluids, 2008, to appear. 